\title{
On the Photographic Reciprocity Law Failure and Related Effects. II. The Low Intensity Sequence Effect
}

\author{
E. KaTZ \\ Physics Department, University of Michigan, Ann Arbor, Michigan \\ (Received October 17, 1949)
}

\begin{abstract}
A quantitative theory of all details of the low intensity sequence effect is given on the basis of the following assumptions. A monatomic Ag speck is unstable against thermal agitation, a diatomic speck is stable, but for development a speck of more than two atoms is required. The theory is based on that for low intensity failure as developed in paper I.
\end{abstract}

\section{INTRODUCTION}

TF a plate is given first an exposure (=intensity $1 \times$ time) of $p E$ with intensity $I_{1}$ and subsequently an exposure $q E=(1-p) E$ with intensity $I_{2}$ it is known that the resulting density $D$, after development etc., will differ from what would have been obtained if the sequence of these two exposures had been reversed. This sequence effect has been studied experimentally for intensities not exceeding the optimum intensity by J. H. Webb and C. H. Evans. ${ }^{1}$ The effect was discovered by Weinland. ${ }^{2}$ P. C. Burton and W. F. Berg who call this the "Weinland effect" have published a series of very interesting studies ${ }^{3}$ in which the effect proves to be a useful tool for obtaining qualitative information concerning the latent image in its first stages of formation (sub-latent image).

The density $D$ as a function of the four independent variables $p, E, I_{1}, I_{2}$ may be represented in a number of ways, for instance by plotting $e \equiv \log E$ as a function of $p$ for constant values of the parameters $D, I_{1}, I_{2}$ (isodense curves) or by plotting $D$ as a function of $p$ for constant values of $E, I_{1}, I_{2}$ (iso-exposure curves). For the purpose of the present paper the isodense plots are the most important ones for reasons given below. Of course any other type of plotting can be converted to the isodense type provided there are sufficient data available.

For both iso-exposure and isodense plots a pair of curves with $p, I_{1}, I_{2}$ and $q, I_{2}, I_{1}$ forms a sequence loop as shown in Fig. 1. In such a loop any pair of points on a vertical line corresponds to sequence reversal. We shall call them conjugate points. Webb's results are mainly given in terms of iso-exposure loops but enough data are available for effectively transforming them into isodense plots. The results of Burton and Berg are given in neither way; these authors mostly keep either $p E$ or $q E$ constant, varying both $E$ and $D$. Not enough data are available for replotting isodense curves from their results. Besides, their papers admittedly do not aim at quantitative relations, for which isodensity is essential, as will be shown below. Rather their results

1 J. H. Webb and C. H. Evans, J. Opt. Soc. Am. 28, 431 (1938)

2 C. E. Weinland, J. Opt. Soc. Am. 16, 295 (1928).

3 P. C. Burton and W. F. Berg, Phot. J. 86B, 2 (1946) and 88B, 84 (1948); P. C. Burton, Phot. J. 86B, 62 (1946) and 88B, 13 and 123 (1948). yield valuable support to the hypotheses concerning latent image formation as postulated previously by Webb and Evans and used extensively in the previous paper. ${ }^{4}$

For the loops obtainable from Webb's data we note three significant features as shown schematically in Fig. 1, viz.:

(a) The loops have a peculiar asymmetric shape.

(b) The curves have flat portions near their $I_{1}$ terminals.

(c) The upper curve in Fig. 1a and the lower one in Fig. $1 \mathrm{~b}$ often have a typical sigmoid bend as they approach the right $I_{2}$ terminal point.

Weinland, and Webb and Evans more fully, recognized qualitatively the relation between the sequence effect and the reciprocity law failure (r.l.f.), in particular the low intensity failure (l.i.f.). The theory of the l.i.f. given in paper I therefore affords a basis for a complete theory of the low intensity sequence effect. The present paper aims at developing this theory and confronting it with Webb and Evans' experimental results.

\section{ELEMENTARY RELATIONS}

After choosing two fixed intensity values $I_{H}$ and $I_{L}\left(I_{H}>I_{L}\right)$ we consider two types of conjugate exposure sequences:
(a) $I_{1}=I_{H} ; \quad I_{2}=I_{L}$
(b) $I_{1}=I_{L} ; \quad I_{2}=I_{H}$.

If a plate is given an exposure $p E$ with $I_{1}$ and $(1-p) E$ with $I_{2}$ the resulting density $D$ is one function of $p$ and $E$ in case $a$ and another function of $p$ and $E$ in case $b$. It will be assumed that the result is independent of the time interval between the end of $I_{1}$ and the beginning of $I_{2}$ and also independent of the time between termination of $I_{2}$ and development. According to Burton and Berg these assumptions are reasonable at low intensities. If required the theory is strictly valid for short interexposure time and short times between exposure and development. Then, for each case, at any point of the $D(p, E)$ surface the following identity holds:

$$
(\partial D / \partial p)_{E}=-(\partial D / \partial \log E)_{p} .(\partial \log E / \partial p)_{D} .
$$

\footnotetext{
4 E. Katz, J. Chem. Phys. 17, 1132 (1949), referred to hereafter
} as "paper I." 
The quantity

$$
\gamma \equiv(\partial D / \partial \log E)_{p}
$$

is the slope of characteristic curves such as shown in Fig. 2. This quantity is practically constant for a large range of intermediate $D$ values. Moreover, for any value of $D, \gamma$ is only very weakly dependent on $p$. Finally $\gamma$ is even to a higher degree of approximation equal for conjugate points, because at the terminals of the loop its two branches necessarily meet. Form. (1) links Fig. 1a and 1b as illustrated in Fig. 2. Where Fig. 1a has a horizontal segment $F$, Fig. 1b must have the same. Insofar as $\gamma$ is constant Fig. $1 b$ is an upside down view of Fig. 1a, apart from a vertical scale factor $\gamma$. This simple rule effects the transformation from isoexposure to isodense curves. The constancy of $\gamma$ for a large $D$ range means that the shape of the sequence loops in Figs. 1a and $1 \mathrm{~b}$ is within the corresponding limits independent of the value of $E$ and $D$.

\section{THE RELATION BETWEEN DENSITOMETRIC AND SINGLE GRAIN DATA}

In order to correlate experimental density measurements with single grain theory we postulate the order principle (o.p.): "The order in which the grains of a given plate become developable in the course of any exposure is independent of the conditions of exposure and development." This postulate is extensively discussed in paper I. A consequence of the o.p. is that isodense exposures produce exactly equal effects in the last grain(s) rendered developable. Deviations from the

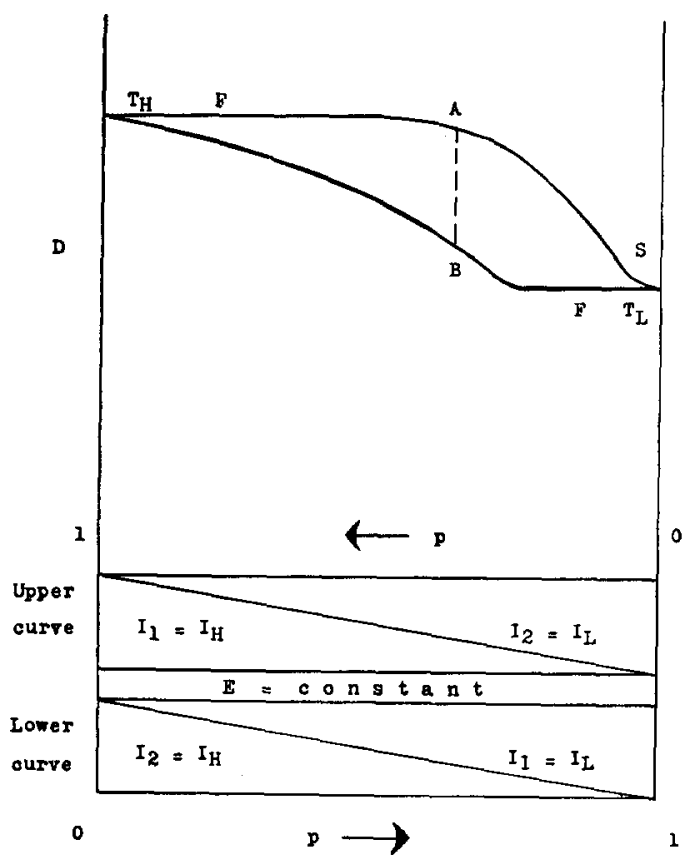

Fig. 1a. Iso-exposure plot. Typical "sequence loops" (schematic for various proportions of exposures at a high intensity $I_{H}$ and at a low intensity $I_{L} . A, B ; A^{\prime}, B^{\prime}=$ conjugate points, $F=$ flat portions; $S=$ sigmoid bend. o.p. and their influence on the present theory will be discussed in Section VIIc.

The o.p. should at this point be compared with the ideas of Burton and Berg. The purpose of this comparison is to show why the present paper is essentially limited to isodense exposures and why, consequently, Burton and Berg's results are not amenable to the same degree of quantitative analysis unless many more details about the grains are known. This can best be seen with the aid of diagrams similar to those designed by Burton and Berg on page 21 of their first paper. We consider a multi-dimensional diagram. The ordinate is the amount of silver $S$ of that speck which is going to be developable first in any particular grain as a result of a certain type of exposure. The abscissa is the total exposure. Along the other axes are plotted all $(n)$ independent parameters of exposure and development. Each grain is then represented by an $(n+1)$-dimensional supersurface $S_{n+1}$. The developability is given by another $(n+1)$-dimensional supersurface $D_{n+1}$ (presumably flat). The $n$-dimensional intersection supercurve of $S$ and $D$ determines the developability of the grain in question. Such a supercurve can in principle be drawn for each grain.

The o.p. may now briefly be stated in terms of this diagram as follows: "Supercurves do not intersect." It has been shown in paper I that the o.p. is the necessary and sufficient condition for establishing a 1-1 relation between single grain data and densitometric measurements and this is exactly what is expressed by the non-intersection of the supercurves. Another

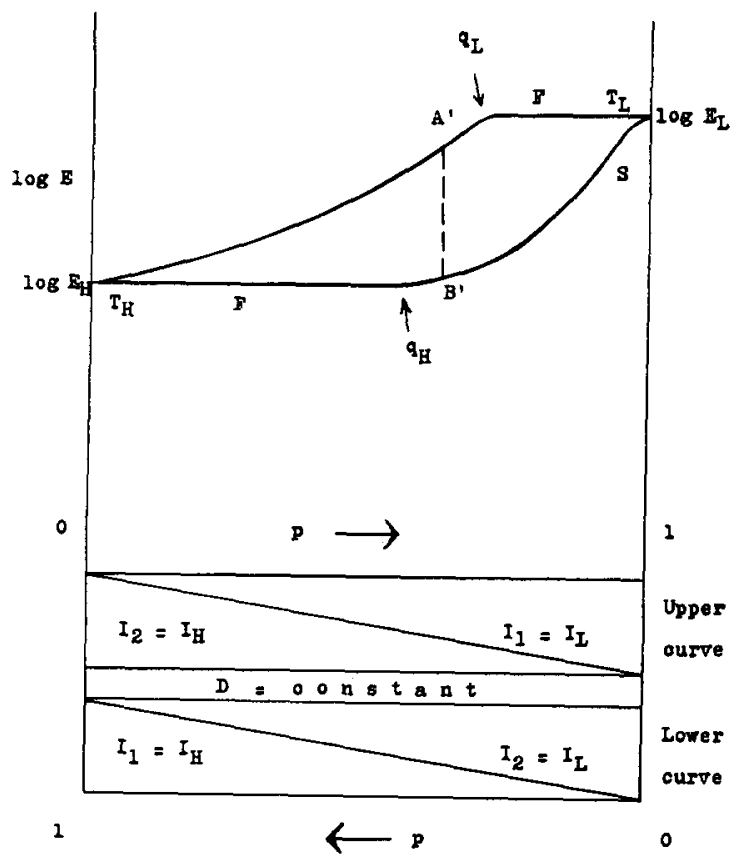

FIg. 1b. Isodense plot. The exposure fraction $p E$ given first at $I_{1}$ (either $I_{H}$ or $I_{L}$ ) is followed by $q E$ at $I_{2}$ (either $I_{L}$ or $I_{H}$ ) $(p+q=1) . q_{L}, q_{H}=$ corners, $T_{L}, T_{H}=$ low and high intensity terminals. 
way of expressing the o.p. is that each supercurve (each grain) may be labeled with the density at which it becomes developable.

By comparing isodense exposures one deals with one and the same grain. One has only to derive the shape of one supercurve. In order to compare non-isodense exposures one has to know, in addition, something about the distances between the supercurves and about the possibilities of intersections of the supersurfaces $S_{n+1}$ at lower levels than $D_{n+1}$. These are governed by grain properties which may differ widely among the various grains of an emulsion even if the comparison is restricted to grains of the same size and at the same depth, as is evidenced for instance by Webb's analysis of $\mathrm{H} \& \mathrm{D}$ curves. $^{5}$ Apart perhaps from very special circumstances, the required details are not known so that in general a quantitative analysis along the ideas of Burton and Berg will not be feasible. Only for isodense exposures where such detailed information is not required may basic single grain theory be expected to succeed.

\section{THE MECHANISM OF THE SEQUENCE EFFECT AND ITS CONSEQUENCES}

The idea of Webb and Evans as corroborated by the work of Burton and Berg is to divide the process of latent image formation into the following two parts: (a) the building of a stable $\mathrm{Ag}$ speck (sub-latent image); (b) the building of a developable speck from a stable one.

Until part $a$ is completed thermal motion will tend to destroy the result. Therefore, the efficiency of this part is sensitive to the temperature and to the light intensity. During part $b$ the efficiency of the process is assumed to be independent of these factors insofar as we restrict ourselves to low intensities. Therefore, changes in intensity during the last part of an exposure will affect the result less than changes during the first part. Qualitatively this picture explains the sequence loops and at the same time the li.f. The terminals of the loops are different because of different efficiencies of part $a$ for different intensities, and this is simply the l.i.f.

The parts $F$ in Fig. 1 are flat because of the constant efficiency of part $b$. Because the flat part of the lower curve in Fig. 1b generally extends to $p>0.5$ Webb and Evans concluded that the size of the stable speck is about half that of the developable speck. No other quantitative conclusions on the subject were drawn in their paper. The interpretation of the length of the flat part was criticized by Burton and will become clear in the present paper.

In paper I extensive use was made of this picture for the theory of the 1.i.f. Likewise we shall assume here that a monatomic Ag speck is unstable against thermal agitation whereas a diatomic one is stable. An additional number of $\mathrm{Ag}$ atoms has to be added in order to reach developability. The efficiency of the process of

\footnotetext{
' J. H. Webb, J. Opt. Soc. Am. 29, 314 (1939).
}

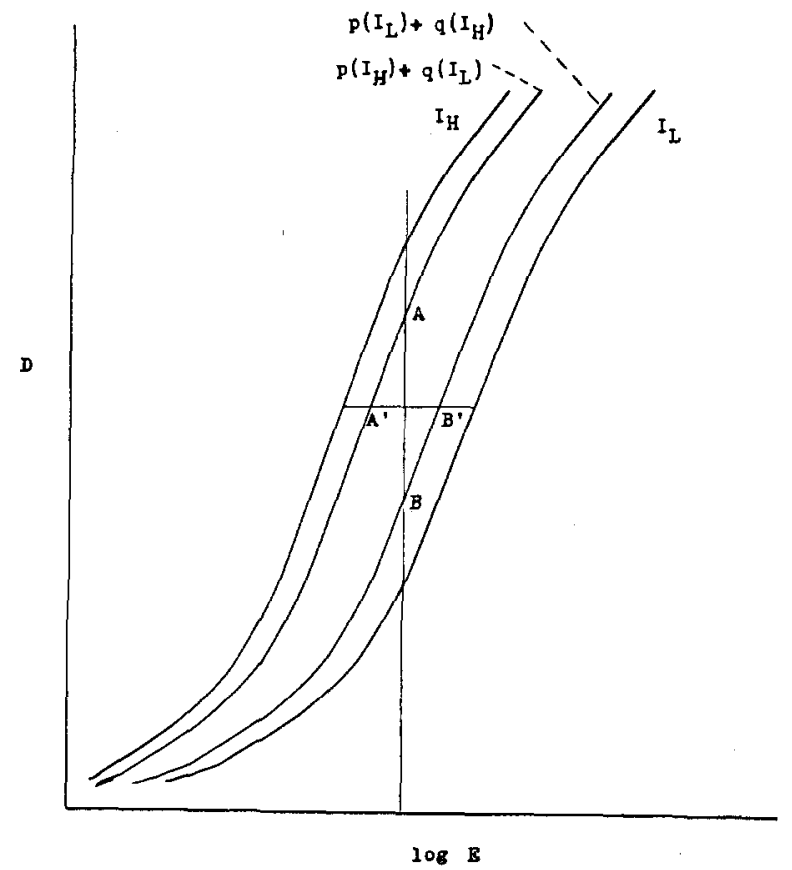

FIG. 2. Schematic representation of terminal characteristic curves and of one pair of conjugate sequence characteristic curves. Vertical section corresponds to iso-exposure diagram Fig. 1a. Horizontal section corresponds to isodense diagram Fig. $1 \mathrm{~b}$.

latent image formation is governed by the time spacings of successive quanta (interquantic times) during the formation of the stable speck.

We wish to find the probabilities that a certain type of exposure will produce in a grain a stable speck or a developable speck. We define the survival function $F(t)$ as the probability that a grain, absorbing at $t=0$ a quantum which produces a conduction electron, will at time $t$ still have this electron "alive." This electron may wander around, being captured and released at intervals, until it finally "dies" either by being trapped stably at a silver speck or by being recaptured in its hole. Let $E . P(T, t) d t$ be the probability of finding in an exposure $E$ of duration $T$ an interquantic time between $t$ and $t+d t$. Let $c$ be the chance of building a stable speck if two electrons are alive. Then the probability of not building a stable speck by two quanta, separated by one interquantic time $t$ is $(1-c F(t))$. The chance $N$ of not building a stable speck by any of the interquantic times occurring in the exposure $E$ is:

$$
\begin{aligned}
N=\prod_{t=0}^{t=\infty}(1-c F(t)) E \cdot P(T, t) d t \\
=\exp \left\{\int_{0}^{\infty} E \cdot P(T, t) \ln (1-c F(t)) d t\right\} .
\end{aligned}
$$

Finally the probability of forming one or more stable specks during $E$ is $1-N$. In these expressions the upper limit of integration may be taken as $T$ or $\infty$ since $P=0$ for $t>T$. We have neglected the probability of a first electron reacting with a third or later one. This 
TABLE I, Parameter values from analysis of Webb and Evans' data.

\begin{tabular}{ccccccc}
\hline Emulsion & $\gamma$ & $\epsilon$ & $\omega$ & $x$ & $p_{H}$ & $\begin{array}{c}\text { Ag atoms } \\
\text { per speck }\end{array}$ \\
\hline II & 4.64 & 2.37 & 3.78 & 0.19 & 0.49 & 4 \\
III & 1.17 & 4.08 & 15.1 & 0.39 & 0.22 & 9 \\
IV & 1.10 & 4.15 & 9.35 & 0.32 & 0.38 & 5 \\
V & 0.76 & 4.60 & 14.7 & 0.39 & 0.26 & 8 \\
II & - & 3.94 & 13.0 & 0.37 & 0.25 & 8 \\
\hline
\end{tabular}

is justified in the low intensity domain we are dealing with here. For the same reason and because $c<1$ we may legitimately break off the series development of $\ln (1-c F(t))$ after the first term in (3) and we find:

$$
\ln N=-c E \int_{0}^{\infty} P(T, t) . F(t) d t \equiv-c E W .
$$

The quantity $W$ defined by this relation is dependent in general on $I$ and $T$. For exposures with many interquantic times $P$, and hence $W$, becomes independent of $T$. For two successive exposures $E_{1}$ with $T_{1}$ and $E_{2}$ with $T_{2}$ a similar reasoning leads to:

$$
\ln N=\ln N_{1}+\ln N_{2}=-c\left(E_{1} . W_{1}+E_{2} . W_{2}\right)
$$

for the probability $N$ that neither exposure will produce a stable Ag speck. We have neglected the probability that the last electron of the first exposure may react with the first one of the second exposure in accordance with the assumption stated in Section II. For many short breaks such as occur in the intermittence effect this probability has to be taken into account (see paper III) but for only one break its influence is calculable but very small.

The probability of forming a developable grain can now be found with good approximation from the previous result by reserving a last part $E_{0}$ of the total exposure $E$ for the purpose of bringing up to or beyond the developable size all specks that were stabilized during $E-E_{0}$ but no specks that were not. In accordance with Webb, Evans, Burton and Berg, we shall take $E_{0}$ to be independent of the intensity at which this part of the process is effected. Owing to the statistical fluctuations of the number of quanta received during $E_{0}$ this procedure is an approximation. The effect of the fluctuations will be estimated in Section VII.

We conclude that the probability for rendering a grain developable is governed by the quantity $Q$ defined as follows:

(a) For an exposure $p E$ at $I_{1}$ followed by $q E \geqslant E_{0}$ at $I_{2}(p+q=1)$

$$
Q=c\left\{W_{1} p E+W_{2}\left(q E-E_{0}\right)\right\} .
$$

(b) For an exposure $p E$ at $I_{1}$ followed by $q E \leqslant E_{0}$ at $I_{2}(p+q=1)$

$$
Q=c . W_{1}\left(E-E_{0}\right)
$$

(c) For only one exposure $E$ at $I$

$$
Q=c \cdot W\left(E-E_{0}\right) \text {. }
$$

Case $c$ can be considered as a special case of $b$ for $q=0$. Assuming the order principle, isodense exposures must correspond to the same probability for developability for the type of grains that become developable last at the density considered. Consequently isodense exposures should satisfy the relation:

$$
Q=\text { constant. }
$$

This then is the general expression for the sequence effect and at the same time for the l.i.f. Its explicit form depends on the $P$ and $F$.

\section{THE STATISTICS OF LIGHT ABSORPTION $P$ AND THE SURVIVAL FUNCTION $F$}

Let a grain absorb $I=1 / \bar{t}$ quanta per second during a time $T$ with $E=I . T$. It is easily shown that the probability $E . P(T, t) d t$ of finding an interquantic time between $t$ and $t+d t$ in $E$ is exactly:

$$
\left.\begin{array}{rlrl}
E . P(T, t) d t & =E(1-t / T) \exp (-t / \bar{t}) d t / \bar{t} & & (0 \leqslant t \leqslant T) \\
P & =0 & (t \geqslant T)
\end{array}\right\} .
$$

For $E \gg 1, P$ approaches Eq. (5) in paper I, becoming independent of $T$. For $E \ll 1$ the only interquantic times that can occur are $\ll \bar{t}$. The number of interquantic times during $E$ is exactly:

$$
E \int_{0}^{\infty} P d t=E-1+e^{-E},
$$

which approaches $E-1$ for large $E$ and $E^{2} / 2$ for small $E$.

The survival function $F(t)$ was discussed in paper $\mathrm{I}$. Its relevant features are: independence of the light intensity in the l.i.f. region, and $F(t) \sim t^{-x}$ for low in-

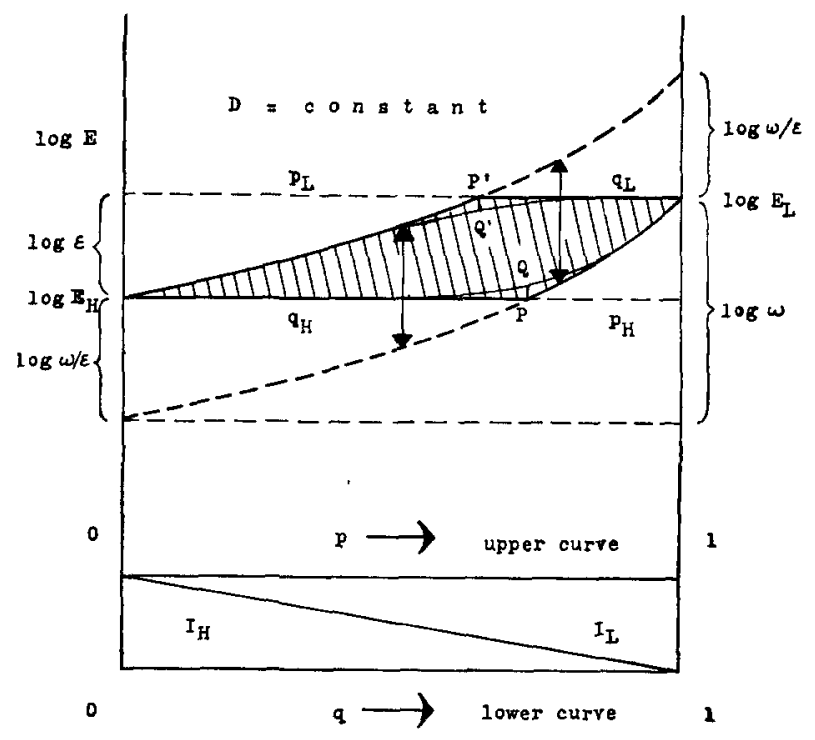

FIg. 3. Schematic illustration of theoretical relations $10 . .16$ for isodense loops. 
tensities. Here $x$ is the slope of the straight part of isodense 1.i.f. diagrams. The indepencence of light intensity amounts to interaction only between successive quanta.

\section{THEORY OF THE ISODENSE LOOPS}

\section{a. First Approximation}

In first approximation $W$ as defined by (4) is assumed to be independent of the exposure time $T$. According to Eqs. (6) and (7) the terminals of the loops should then satisfy:

$$
W_{H}\left(E_{H}-E_{0}\right)=W_{L}\left(E_{L}-E_{0}\right)
$$

and the loops are given by:

$$
W_{1}\left(E-E_{0}\right)=W_{H}\left(E_{H}-E_{0}\right)
$$

if $q E \leqslant E_{0}$ and by:

$$
W_{1} p E+W_{2}\left(q E-E_{0}\right)=W_{H}\left(E_{H}-E_{0}\right)
$$

if $q E \geqslant E_{0}$.

The meaning of the symbols is defined in Section II. Equation (11a) represents the flat parts $F$. The upper and lower branch correspond, respectively, to $I_{1}<I_{2}$ and $I_{1}>I_{2}$. At the points $q_{H}=E_{0} / E_{H}$ and $q_{L}=E_{0} / E_{L}$ the curves have a discontinuous slope, caused by the discontinuous change from unstable to stable specks and the neglection of statistical fluctuations in the number of quanta absorbed during $E_{0}$. The different lengths of the flat parts of the two branches, which contribute to the asymmetry of the loop are now seen to be connected with the difference of $E_{H}$ and $E_{L}$.

We abbreviate

$$
\begin{aligned}
W_{H} / W_{L} & =\omega \\
E_{L} / E_{H} & =\epsilon,
\end{aligned}
$$

where $\omega$ and $\epsilon$ are $>1$.

According to paper $I$, for low intensities, $W$ is proportional to $I^{x}$ where $x$ is the l.i.f. exponent. Since the bending of the li.f. curve towards the optimum was primarily due to the factor $E-E_{0}$ we shall assume the validity of this relation up to the optimal intensity. A glance at Fig. 5 on p. 129 of Burton's last-mentioned paper supports the correctness of this assumption. Then:

$$
\omega=\left(I_{H} / I_{L}\right)^{x} .
$$

If is seen that for given intensities the knowledge of the 1.i.f. yields directly $\epsilon$ and $\omega$. It will be shown that these two parameters determine the shape of the loops completely. By straightforward manipulation of Eqs. (10), (11), and (12) the equations of the curved parts of the isodense loop become:

upper branch :

$$
\log E=\log E_{H}-\log (1-p(1-1 / \omega))
$$

lower branch:

$$
\log E=\log E_{H}-\log (1-q(1-1 / \omega))+\log \epsilon / \omega .
$$

Since $p$ for the upper branch and $q$ for the lower branch both run from left to right in Fig. 1b the two curves have exactly the same shape and differ only by a vertical sift of

$$
\Delta \log E=\log \omega / \epsilon .
$$

The shape of the curves is only dependent on one parameter $\omega$. These results are illustrated schematically in Fig. 3.

The following relations are helpful in the analysis of experimental curves and are easily verified:

$$
\begin{aligned}
q_{H} / q_{L} & =\epsilon, \\
q_{H} p_{L} / q_{L} p_{H} & =\omega,
\end{aligned}
$$

from which the $p$ 's and $q$ 's may be derived separately.

The area of the loop can also be computed. It approaches zero if the two intensities are very low, because $\omega \rightarrow \epsilon$, and also if the two intensities are near the

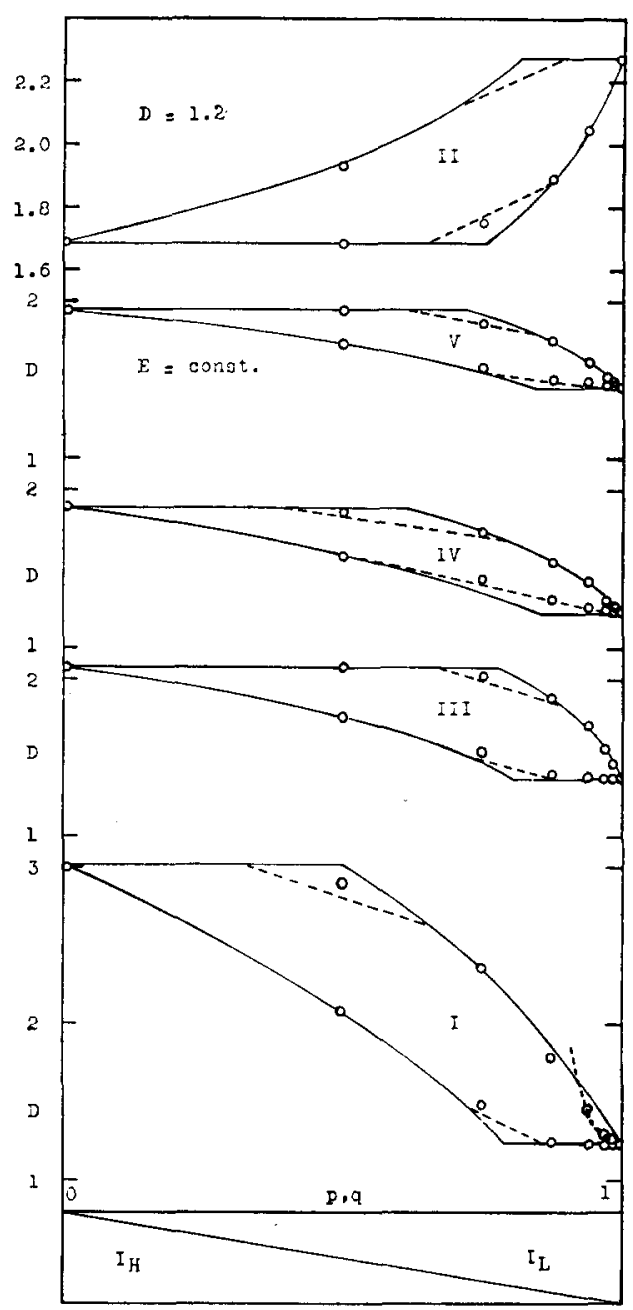

Fig. 4. Comparison of theory with Webb and Evans' experimental results (reference 1). Points are experimental. Full lines are drawn according to the theory in first approximation. Dotted lines represent estimated second approximation. Top loop for emulsion II: isodense plot. Other loops are iso-exposure plots. Used values of the constants are listed in Table I. 
optimal region, because $\epsilon \rightarrow 0$. We find

$$
\text { area }=0.434(\omega-\epsilon)(\epsilon-1) /(\omega-1) \epsilon,
$$

which is maximum for

$$
\epsilon=\omega^{0.5}=\left(I_{H} / I_{L}\right)^{x / 2},
$$

in which case the corners are conjugate points. This condition was intuitively satisfied remarkably closely by Webb and Evans (see Table I).

\section{b. Comparison of First Approximation with Experiment}

The lower part of Fig. 4 shows Webb and Evans' measured points for their emulsions I, III, IV, V. Here only iso-exposure data were available, so that the theory has three adjustable parameters: $\gamma, \omega$, and $\epsilon$. The full curves give the lines according to (14) transformed to iso-exposure plots according to Section II. The values of $\gamma, \omega$, and $\epsilon$, given in Table $I$, have been derived from the following sets of three data:

(a) The vertical distance between the terminals $\gamma \log \epsilon$.

(b) The vertical distance between the curves $\gamma \log \omega / \epsilon$ (see (15) and Fig. 3).

(c) The slope at the low intensity terminal 0.434 $\gamma(\omega-1)$, disregarding the sigmoid bend if present.

A more stringent test is possible for emulsion II, for which Webb and Evans, in their Figs. 4 and 5, give enough information for plotting a number of isodense points. Here the theory has only two adjustable parameters:

(a) The vertical distance between the terminals $\log \epsilon$.

(b) The slope at the low intensity terminal 0.434 $(\omega-1)$.

The results of Fig. 4 show that a satisfactory description is achieved with reasonable values of the parameters $\gamma, \omega, \epsilon$. The values of the l.i.f. slope $x$ that follow with (13) from $\omega\left(I_{H} / I_{L}\right.$ being 1000) are tabulated and also turn out reasonable. In particular emulsion I which according to reference 1 has very low speed and thus probably fine grains, has a lower $x$-value in agreement with the rule found in paper $I$.

In two respects systematic deviations appear between the points and lines. The corners are actually rounded and the sigmoid bend $S$ (Fig. 1) is ignored. These features will be cleared satisfactorily in the second approximation.

In order to interpret the length of the flat part $q_{H}$ he following relation is useful:

$$
1 / p_{H}=E_{H} /\left(E_{H}-E_{0}\right) .
$$

If one assumes that for optimal intensity the stabilation of the Ag speck occurs with the same efficiency ' the further growth of the stable speck, then accordr to (19) the value of $1 / p_{H}$ for $I_{H}=I_{\text {opt }}$ should be integral multiple of 0.5 , assuming a diatomic speck be the smallest stable one. The permissible values of
TABLE II. Rounding of corners resulting from various causes. Values of $P Q$ and $P^{\prime} Q^{\prime}$ for emulsions II in units of $\log E$, others in units of $D$.

\begin{tabular}{ccccccc}
\hline \hline & \multicolumn{2}{c}{ Fluct. $E_{0}$} & & & \multicolumn{2}{c}{ Resultant } \\
Emuls. & $P Q$ & $P^{\prime} Q^{\prime}$ & $P Q=P^{\prime} Q^{\prime}$ & $\begin{array}{c}\text { Grain size } \\
P Q=P^{\prime} Q^{\prime}\end{array}$ & $P Q$ & $P^{\prime} Q^{\prime}$ \\
\hline I & 0.24 & 0.12 & 0.02 & 0.03 & 0.24 & 0.12 \\
II & 0.09 & 0.03 & 0.05 & 0.06 & 0.10 & 0.07 \\
III & 0.13 & 0.04 & 0.05 & 0.06 & 0.13 & 0.07 \\
IV & 0.12 & 0.06 & 0.04 & 0.05 & 0.12 & 0.07 \\
V & 0.08 & 0.02 & 0.05 & 0.06 & 0.09 & 0.06 \\
\hline \hline
\end{tabular}

$p_{H}$ are then: $0.67 ; 0.50 ; 0.40 ; 0.33 ; 0.285 ; 0.25 ; 0.22$; ..., corresponding to a developable speck of $3,4,5$, 6 ... Ag atoms. Only the first four or so values are sufficiently separated for checking the assumption concerning the efficiency experimentally. The value of $p_{H}=0.50$ apparently was taken by Webb and Evans as an average value but the present analysis proves, as shown in Table I that also other values occur. So far the observed values are close enough to the permitted ones not to contradict the assumption made. The number of atoms per just developable Ag speck corresponding to these $p_{H}$ values are also listed.

\section{SECOND APPROXIMATION}

The theory needs refinement in three ways: (a) The dependence of $W$ on $E$ should be taken into account, especially for small values of $E$. (b) The statistical fluctuations in the number of quanta received during the last part of the exposure $E$ must be taken into account. (c) Deviations from the order principle, resulting from a light gradient in the plate and inhomogeneous grain size, have to be analyzed. The correction under (a) will explain the sigmoid bend, whereas the other two causes round off the corners of the curves.

\section{a. The Dependence of $W$ on $E$}

The flat parts are obviously not affected by any dependence of $W$ on $E$. The effect for the upper curve left terminal (Fig. 3) turns out to be negligible. The discussion may thus be restricted to the lower curve right terminal.

For small values of $p$ the first term in (11b), in view of (8) and (4) becomes $(p E)^{2} / 2$. The second term is not altered. The resulting equation for the isodense curve near the terminal is

$$
\log E / E_{L}=0.434\left(p-\epsilon \omega E_{H} p^{2} / 2 W_{H} \ldots\right),
$$

whereas $(14 \mathrm{~b})$ originally would yield

$$
\log E / E_{L}=0.434\left(-p(\omega-1)+p^{2}(\omega-1)^{2} / 2 \ldots\right) .
$$

Equation (20) represents the sigmoid bend. The horizontal distance between the two curves (20) and (21) has a maximum of

$$
\Delta p_{\max }=0.5 \omega^{2} /(\omega-1)\left((\omega-1)^{2}+\epsilon \omega E_{H} / W_{H}\right)
$$

around the point

$$
p=\omega /\left((\omega-1)^{2}+\epsilon \omega E_{H} / W_{H}\right) .
$$


Using the values for $\epsilon$ and $\omega$ from Table I it is seen that emulsion I can be fitted near the terminal by (20) with $E_{H} / W_{H}=10$ (dotted curve in Fig. 4). With the observed $q_{H}=0.5$ one finds with (10) $E_{H} W_{H}=4$ or $W_{H}=0.65$ and $E_{H}=6.5$. These values are reasonable. In general $W_{H \text {.opt }}$ must be less than, but of the order of unity. Using the same value of $E_{H} / W_{H}$ also for the other emulsions one finds $\Delta p_{\max }=0.01$. This is almost unobservably small, showing the reason why the other curves have, if anything, only a trace of this bend.

\section{b. The Statistical Fluctuations of $E_{0}$}

In Eq. (11) $E_{0}$ represents the exposure, required for bringing a just stable speck to the just developable size. This requires a constant number of quanta for the grains that become developable last. Hence the magnitude of $E_{0}$ that will furnish these quanta fluctuates statistically about some average value $E_{a}$. The influence of these fluctuations is to round off the curves at the sharp corners resulting from the first approximation (see Fig. 3). The curves for grains with various values of $E_{0}$ coincide along the horizontal parts since $\epsilon$ is independent of $E_{0}$, but their corners occur at different values of $q$ which is proportional to $E_{0}$ according to (11). We shall measure the degree of rounding of the corner by the vertical distance $P Q$ between the corner of the simple curve corresponding to $E_{a}$ and the resultant curve. The distribution of $E_{0}$ is given by

$$
P\left(E_{0}\right) d E_{0}=E_{0}{ }^{E_{a}-1} e^{-E_{0}} /\left(E_{a}-1\right) ! d E_{0} .
$$

From (22) $P Q$ can be estimated. It is found that $Q$ at $q_{H}$ must lie on a curve with an $E_{0}$ value about $10-20$ percent lower than the $E_{a}$ value defining $P$. The percentage depends very insensitively on $E_{a}$, being lower if $E_{a}$ and $q_{H}$ are larger.

In view of (16a) $P^{\prime} Q^{\prime}$ for the $q_{L}$ corner must be of the order $\epsilon$ times smaller than $P Q$ at the $q_{H}$ corner. The actually observed rounding of the $q_{L}$ corner is much greater than this amount and is due to other causes discussed under $c$. The roundings estimated in this way are shown in Table II.

\section{c. Deviations from the Order Principle}

Deviations from the o.p. may result from the light intensity gradient in the plate and from the grain size inhomogeneity. Their effects can be treated, as outlined in paper I, by considering the emulsion as a sum of fractions, indicated by a parameter $\alpha$ such that in each fraction the o.p. holds. The total density is then the sum of all fractional densities

$$
D=\int D(\alpha, e, p) d \alpha,
$$

where $e=\log$ (incident exposure on the plate). Applying the requirement of isodensity $\delta D=0$, setting

$$
(\delta D(\alpha, e, p) / \delta e)_{p}=\gamma_{\alpha}
$$

and noticing

$$
\int \gamma_{\alpha} d \alpha=\gamma
$$

We find with (1)

$$
\partial e / \partial p=1 / \gamma \cdot \int \gamma_{\alpha}(\partial e / \partial p)_{D} d \alpha=(\partial e / \partial p)_{\text {Av } \cdot \alpha} .
$$

I.e. the slope of the sequence loop at any point $p$ is the average slope at that point for the various $\alpha$-groups. We have to analyze now what causes spreading values of the slope $\partial e / \partial p$.

For the intensity gradient deviations from the o.p. the l.i.f. exponent $x$, hence $\omega$ is not dependent on $\alpha$, but $\epsilon$ is. $E_{H}$ is practically independent of $\alpha$ but $\log E_{L}$, and hence $\log \epsilon$, varies as $x \log I$. For the grain size deviations from the o.p. it has been shown in paper I that $x$, hence $\omega$, may depend very slightly on $\alpha$ but $\epsilon$ is much more affected, mainly through variations in the rate of quanta reception that go with variations in grain size. Practically then for both types of deviations $\omega$ is constant and $\epsilon$ varies. Since $\omega$ determines the shape of the curved parts of the loop and $E_{H}$ is unaffected the entire left half of the loop is practically without spread. The right half has a vertical spread, given by the mean absolute deviation $\Delta$ in $\log \epsilon$. The resulting roundings of the upper and lower corners, $P Q$ and $P^{\prime} Q^{\prime}$, are now both equal to $\Delta / 2$. Values of $\Delta$ can be estimated in the following way.

For the intensity drop through the plate a factor of 10 is a commonly occurring order of magnitude. The mean absolute spread in $\log I$ is then 0.25 hence $P Q=P^{\prime} Q^{\prime}=0.125 x$. For the mean absolute grain size spread, according to paper I, a common order of magnitude is $0.8\left((\Delta \log \alpha)_{\text {Av }}^{2}\right)^{0.5}=0.32$. This is effectively an intensity spread and hence $P Q=P^{\prime} Q^{\prime}=0.16 x$. A little geometrical consideration will show that the combination of several spreading causes results in a lengthening of the rounded section, leaving the height $P Q$ practically equal to the greatest partial $P Q$ value of the separate causes. The effects of the various spreads are collected in Table II. They should be interpreted only as a rough estimate because of the unknown exact values that go into them. The dotted lines in Fig. 4 are drawn according to the resultant values of the table. The agreement with the experimental points is remarkably good. It is seen that the rounding of $q_{H}$ is mainly determined by the fluctuations of $E_{0}$, whereas that of $q_{L}$ comes mainly from the grain size spread except for emulsion I.

It is noteworthy that the theory of the loops at this point permits ruling out the hypothesis mentioned in paper $\mathrm{I}$, that the l.i.f. slope $x$ should be the result of the combination of two groups of grains, one group with slope 0 and the other with slope 1. According to Eq. (21) the slope of the curved part near the low intensity terminal, disregarding the $S$ shape, is $0.434(\omega-1)$ 
where $\omega=1000^{x}$. The slope would be 0 for the first group and 434 for the second one. The average for $\bar{x}_{A v}=0.33$, according to (24) would be about 150 . This is incompatible with the observed slopes of about 5 . The basic idea of this argument is that the r.l.f. averages $x$ and the terminal slope of the loops averages $(1000)^{x}$; these two averages are quite different. This argument, therefore, proves again the basic idea of paper I, that the value of $x$ observed is essentially a single grain feature.

\section{CONCLUSION}

The theoretical considerations in this paper yield these conclusions: (1) The available data for isodense and iso-exposure sequence loops are described quantitatively by the theory in terms of l.i.f. The latter in turn was covered in paper $I$ in terms of the fundamental processes in the grain. (2) The number of Ag atoms in a just developable speck seems to vary somewhat according to circumstances between 4 and 10. (More data may reveal even larger values.)

\footnotetext{
THE JOURNAL OF CHEMICAL PHYSICS

VOLUME 18 , NUMBER 4

A P R I L , 1950
}

\title{
The Raman Spectrum of Hexachlorodisilane
}

\author{
Mikio Katayama, Takehiko Stmanouti, Yonezo Morino, and San-Ichiro Mizushima \\ Chemical Laboratory, Tokyo University, Bunkyo-ku, Tokyo, Japan
}

(Received October 13, 1949)

\begin{abstract}
The Raman spectrum of hexachlorodisilane was observed in the liquid state and the normal vibrations of this molecule were calculated by use of the Urey-Bradley field. The seven observed Raman frequencies could be assigned to the fundamental vibrations and the molecule was found to belong to the $D_{3 h}$ ' symmetry. This means that the molecule exerts internal free rotation around the Si-Si bond as axis. This is in conformity with the experimental result of the electron diffraction investigation.
\end{abstract}

D URING the last few years many investigations of the molecules of ethane type have been made in connection with the mutual position of the two methyl groups and the potential barrier restricting internal rotation about the carbon-carbon single bond as axis. It would be interesting from this point of view to study the internal rotation of hexachlorodisilane $\mathrm{Cl}_{3} \mathrm{Si}-\mathrm{SiCl}_{3}$ about the $\mathrm{Si}-\mathrm{Si}$ single bond, whose length is much greater than that of the $\mathrm{C}-\mathrm{C}$ bond, so that the interaction potential of the two rotating groups would decrease considerably. L. O. Brockway and J. Y. Beach ${ }^{1}$ concluded by electron diffraction investigation that this molecule has a staggered form, but recently more accurate analysis of the electron. diffraction pattern showed that this molecule exerts almost free internal rotation about the $\mathrm{Si}-\mathrm{Si}$ axis. ${ }^{2}$

F. Stitt and D. M. Yost ${ }^{3}$ observed the frequencies, relative intensities and depolarization factors of the Raman lines of this molecule, but they could not decide whether the molecular configuration corresponds to $D_{3 d}$ or $D_{3 h^{\prime}}$. It is the object of this paper to determine the configuration of this molecule by the measurement of the Raman effect together with the calculation of normal vibrations.

\section{EXPERIMENTAL}

The apparatus for the measurement of the Raman effect was previously described. ${ }^{4}$ The hexachlorodisilane

${ }^{1}$ L. O. Brock way and J. Y. Beach, J. Am. Chem. Soc. 60, 1836 (1938).

2 Yamasaki, Kotera, Tatematsu, and Iwasaki, J. Chem. Soc. Japan 69, 104 (1947).

${ }^{3}$ F. Stitt and D. M. Yost, J. Chem. Phys. 5, 90 (1937).

${ }^{4}$ S. Mizushima and Y. Morino, Proc. Ind.Acad. Sci.8,351 (1938). (b.p. $36.0^{\circ} \mathrm{C}$ at $10 \mathrm{~mm} \mathrm{Hg}$ ) kindly prepared by Professor $\mathrm{K}$. Yamasaki by the reaction between metallic silicon and chlorine gas ${ }^{2}$ was distilled into the Raman tube in vacuum. To avoid the decomposition of the sample by ultraviolet rays dilute solution of sodium nitrite was used as a filter. The observed Raman frequencies $(\Delta \nu)$ and visually estimated intensities $(I)$ are shown in Table I, which agree well with the results obtained by Stitt and Yost. The minor differences are that we observed one broad line at $379 \mathrm{~cm}^{-1}$ instead of the two lines at $377 \mathrm{~cm}^{-1}$ and $387 \mathrm{~cm}^{-1}$ and that we assigned the $e-565 \mathrm{~cm}^{-1}$ line reported by them as $f-622$ $\mathrm{cm}^{-1}$.

\section{CALCULATION OF NORMAL VIBRATION AND ASSIGNMENT OF FREQUENCIES}

This molecule consisting of eight atoms has twelve normal frequencies, of which six correspond to doubly degenerate vibrations. One of these corresponds to the internal rotation of $\mathrm{SiCl}_{3}$ groups about the $\mathrm{Si}-\mathrm{Si}$ bond as axis and is inactive both in the Raman effect and the infra-red. The selection rule for the remaining eleven vibrations is shown in the left part of Table II, from which we see that six fundamental frequencies will be observed in the Raman effect if the molecule belongs to $D_{3 d}$ symmetry (staggered form), while nine frequencies will be expected both for $D_{3 h}$ (eclipsed form) and $D_{3 h}{ }^{\prime}$ symmetry (free rotation). Stitt and Yost considered that one cannot discriminate $D_{3 h}{ }^{\prime}$ from $D_{3 d}$ by the Raman data, because the frequencies of $E_{g}$ vibrations coincide with those of $E_{u}$ vibrations so that only six fundamentals become Raman active in both cases. Their reasoning is not, however, sound, since it 